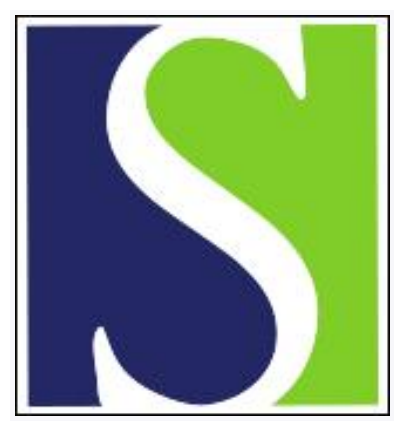

Scand J Work Environ Health 2010;36(3):266-267

https://doi.org/10.5271/sjweh.2903

Published online: 19 Feb 2010, Issue date: 01 May 2010

Occupational rhinosinusitis due to etoposide, an antineoplastic agent

by Meyer HW, Stahl Skov P

Affiliation: Clinic of Occupational Medicine, Hillerød Hospital, 3400 Hillerød, Denmark. hmeyer@dadlnet.dk

Key terms: allergy; antineoplastic agent; case report; cytostatica; etoposide; histamine; histamine release; histamine release test; nurse; occupation; occupational rhinosinusitis; rhinosinusitis

This article in PubMed: www.ncbi.nlm.nih.gov/pubmed/20174772

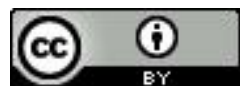




\title{
Occupational rhinosinusitis due to etoposide, an antineoplastic agent
}

\author{
by Harald W Meyer MD, PhD, 1, 2 Per Stahl Skov, MD, DMSc, ${ }^{3,4}$
}

\begin{abstract}
Meyer HW, Stahl Skov P. Occupational rhinosinusitis due to etoposide, an antineoplastic agent. Scand J Work Environ Health. 2010;36(3):266-267.

Objective This paper reports a rare case of an occupational hypersensitivity reaction to an antineoplastic agent

Methods This is a clinical case report of a 45 -year-old nurse who developed throat irritation and chronic nasal congestion followed by sinusitis shortly after beginning work at an oncological out-patient clinic. The symptoms disappeared upon leaving the clinic two years later, but they returned when she resumed work at the oncology unit at Hillerød Hospital, Denmark, handling chemotherapy on a daily basis. We performed in vitro histamine release tests against nine suspected antineoplastic agents.
\end{abstract}

Results The patient's histamine release test against the antineoplastic agent etoposide was positive; the other test results were negative. The histamine release test against etoposide using passive sensitization was also negative. Upon leaving the oncology department, the symptoms of the nurse disappeared once again. She was given a diagnosis of rhinosinusitis.

Conclusion This case of a hypersensitivity reaction to etoposide was judged to be of occupational origin. It was not clear whether it was immunoglobulin E ( $\operatorname{IgE})$ or non- $\operatorname{IgE}$ mediated.

Key terms allergy; cytostatica; histamine release test; nurse; occupation.

This paper presents a case of a 45-year-old nurse with ten years of work experience. She was first appointed to work in the oncology department of an out-patient clinic in 2004. She treated six cancer patients per day, giving 10-15 chemotherapy infusions. She estimated that she gave etoposide infusions three times a week. In the treatment room, nine cancer patients were treated at a time, and an etoposide cure was given somewhere in the room approximately 2-3 times a day.

Shortly after commencing employment, the nurse developed throat irritation and chronic nasal congestion, followed by sinusitis that was treated with penicillin. Her symptoms reappeared with increasing frequency during the rest of her two-year appointment. In the following year, working away from oncology, her symptoms disappeared, but returned when she was once again employed in an oncology unit, this time at our hospital, Hillerød Hospital. During the first three months working in the ward, no symptoms appeared. However, after only a few days at the out-patient clinic, where she handled chemotherapy treatments daily, the well-known symptoms returned. Ten patients were typically treated at a time in the out-patient room. Here she estimated that the staff delivered three cures of etoposide on a daily basis; on average, she herself gave one of these infusions. In addition, she gave approximately nine other types of chemotherapy treatments per day.

As a result of the rhinosinusitis, she went on shortterm sick leave and after returning to the ward, where she gave only one daily infusion of chemotherapy, her symptoms decreased. At the time of the first consultation at our clinic three months later, she had no more symptoms. There was no clear pattern of her symptoms worsening when giving a particular antineoplastic agent, and she actually suspected two other neoplastic agents to be the cause.

1 Clinic of Occupational Medicine, Hillerød Hospital, Hillerød, Denmark.

2 Department of Occupational and Environmental Medicine, Bispebjerg University Hospital, Copenhagen, Denmark.

3 Allergy Clinic, National University Hospital of Copenhagen, Copenhagen, Denmark.

4 Allergy Centre, Odense University Hospital, Odense, Denmark. 


\section{Methods}

For ethical reasons, a controlled exposure to the handled cytostatica could not be performed. Due to toxicity, an allergic reaction to the antineoplastic drugs could not be verified by a skin prick test, and commercial specific immunoglobulin $\mathrm{E}$ ( $\operatorname{IgE}$ ) tests were not available. An in vitro histamine release test was, however, an alternative (1). A blood sample was therefore incubated with the suspected drugs (etoposide, taxoterere, CTX, 5-FU, oxaliplatin, carboplatin, topotecan, farmorubicin, and gemcitabin) each in 12 concentrations. To evaluate a possible IgE-mediated reaction, we incubated stripped donor basophils with patient serum and, thereafter, the passively sensitized cells with the etoposide. This serum method has previously verified IgE-mediated chlorhexidine allergic reactions (1).

\section{Results}

As the only positive test result, a positive histamine release in the patient's blood was found for etoposide at 10 and $3 \mu \mathrm{g} / \mathrm{ml}$ - in contrast to the results of four healthy volunteers, which were all negative. We did not find a significant histamine release to etoposide in the patient's serum using the passive sensitization method. There might be several explanations for this: (i) a low level of etoposide-specific $\operatorname{IgE}$ was not sufficient to sensitize IgE-stripped donor basophils in the indirect histamine release method; (ii) the reaction is non-IgE-mediated, where etoposide might be a weak histamine releaser activating only hyperreactive basophils from this patient, as demonstrated earlier in persons reacting to perfume (2); or (iii) etoposide might activate other cells in the blood releasing substances with histamine releasing potential.

Despite the positive etoposide histamine release test, it was not possible to distinguish the pathophysiological mechanism as being IgE or non-IgE mediated.

Antineoplastic agents are excreted in body fluids including sweat, and employees can be exposed both via skin contact and lungs (dust/aerosols). Both from 2004-2006 and again in 2007, the antineoplastic agents were delivered ready for infusion and sealed in plastic bags, so the nurse only connected the infusion and took part in normal care of the patients. There was no history of accidental skin exposure to high concentrations of cytostatica. Gloves and a plastic apron were used when handling the infusion kits or when there was a risk of exposure to body fluids or excretions. According to safety instructions, airway protection equipment was not worn. The main exposure was, therefore, most likely to have been secondary from the patients in the form of aerosols or dust that the nurse inhaled.
It is well known that patients treated with etoposide can develop hypersensitivity reactions (3). To our knowledge, occupational hypersensitivity to etoposide among hospital staff has not been reported before. There is one report from Poland of occupational asthma due to another antineoplastic drug, mitoxantrone, confirmed by bronchial provocation and bronchoalveolar lavage (4).

\section{Concluding remarks}

This case of rhinosinusitis was judged to be of occupational origin. It is not clear whether it was $\operatorname{IgE}$ or non-IgE mediated.

Considering this appears to be only the second case reported worldwide, the occupational protection level seems to be appropriate with respect to hypersensitivity. On the other hand, there are reports of other symptoms among nursing staff in oncology wards (5), and significant increases in genotoxicological assessments compared to non-exposed control groups have also been reported $(6,7)$. So, for several reasons, it is still important to maintain a high protection level against antineoplastic drugs among nursing and cleaning staff at oncological departments.

\section{References}

1. Garvey LH, Kroigaard M, Poulsen LK, Skov PS, Mosbech H, Venemalm L, et al. IgE-mediated allergy to chlorhexidine. J Allergy Clin Immunol. 2007;120(2):409-15.

2. Elberling J, Skov PS, Mosbech H, Holst H, Dirksen A, Johansen JD. Increased release of histamine in patients with respiratory symptoms related to perfume. Clin Exp Allergy. 2007;37(11):1676-80.

3. Collier K, Schink C, Young AM, How K, Seckl M, Savage P. Successful treatment with etoposide phosphate in patients with previous etoposide hypersensitivity. Oncol Pharm Pract. 2008;14(1):51-5.

4. Walusiak J, Wittczak T, Ruta U, Palczynski C. Occupational asthma due to mitoxantrone. Allergy. 2002;57:461.

5. Krstev S, Perunicić B, Vidaković A. Work practice and some adverse health effects in nurses handling antineoplastic drugs. Med Lav. 2003;94(5):432-9.

6. Jakab MG, Major J, Tompa A. Follow-up genetoxocological monitoring of nurses handling antineoplastic drugs. J Toxicol Environ Health Part A. 2001;62(5):307-18.

7. Cornetta T, Padua L, Testa A, Ievoli E, Festa F, Tranfo G, et al. Molecular biomonitoring of a population of nurses handling antineoplastic drugs. Mutat Res. 2008;638(1-2):75-82.

Received for publication: 14 October 2009 